\title{
LEITARTIKEL
}

\section{Alpha Heavy Chain Disease}

\author{
Maxime Seligmann
}

Alpha chain disease ( $\alpha \mathrm{CD}$ ) is the most frequent disorder among the group of heavy chain diseases. Since its first description in $1968 \propto$ CD has been recognized in about 80 patients to our knowledge.

Alpha chain disease is defined by the production of a homogeneous population of molecules devoid of light chains and consisting of incomplete $\alpha$ heavy chains. The $\alpha$ CD proteins are all related to only one subclass of $\alpha$ chains. All 50 proteins which have been typed belonged to the $\alpha 1$ subclass.

The molecular weight of the basic monomeric polypeptide subunit was found to vary between 29,000 and 34,000 i.e. greater than half and smaller than $3 / 4$ of normal $\alpha 1$ chains. Antigenic analysis and preliminary chemical studies indicated that the entire Fc fragment was present in $\alpha$ CD proteins, that their carboxy terminus was identical to that of normal $\alpha 1$ chains and that the heavy-light peptide was missing. The hinge region was shown to be present. In view of these results, the missing portion of the chain is located in the $\mathrm{Fd}$ segment and involves both the $\mathrm{V}$ and $\mathrm{C} 1$ regions. The $\mathrm{N}$ terminal residues of several $\alpha \mathrm{CD}$ proteins were shown to be heterogeneous and attempts obtain the $\mathrm{NH}_{2}$ terminal sequence on an automated sequencer were unsuccessful. The most likely explanation of this heterogeneity of the $N$ terminus is that it is the consequence of a post-synthetic proteolytic process which takes place presumably at the intracellular level. Careful cellular biosynthetic studies performed in one patient excluded the possibility of the synthesis of an entire normal size $\alpha$ chain which would be sequentially degraded to a smaller fragment after its release from the ribosomes. Structural studies were performed on the protein of the same patient. The comparison between the amino acid sequence of the hinge region of this protein and that of a normal IgA1 showed that, after a short segment corressponding to the variable region, it displays a gap which comprises almost the whole Fd segment including the $\mathrm{CH} 1$ constant domain. Normal synthesis resumes at a Valine residue in the hinge region and from there on, the molecule is apparently normal. This XCD protein is therefore synthesized as an internally deleted $\alpha 1$ heavy chain. Thus the primary defect in $\alpha$ CD proteins appears to be a deletion affecting in both instances the variable and first constant regions of the heavy chains, which are under independent genetic control. Any genetic hypothesis about $\alpha \mathrm{CD}$ proteins

Eingegangen am 28. 5. 1975. 
should also take into account the fact that the absence of light chains is due to a lack of synthesis. Since light and heavy chains are under the control of non-linked genes, this peculiar situation raises a puzzling problem for the cellular geneticist. Whether $\alpha$ CD proteins should be considered as "abnormal" is still open to discussion. It should be emphasized that heavy chain disease proteins represent striking (and relatively easily detectable) examples of a less rare event, i.e. the occurence of deletions in myeloma proteins. Various types of heavy and/or light chain deletions have now been described in human and murine monoclonal immunoglobulins.

The diagnosis of $\alpha \mathrm{CD}$ relies entirely upon immunochemical analysis of the serum proteins. It can easily be missed on the serum electrophoretic pattern. When detectable by this procedure, the $\alpha$ CD pathological proteins show an abnormal broad band usually in the $\alpha 2$ and $\beta$ regions. The characteristic sharp peak which is suggestive of a monoclonal type of Ig abnormality is always lacking. Moreover, in half of the cases, the pathological protein is not noticeable on the electrophoretic pattern. The striking and unexpected electrophoretic heterogeneity of these presumably monoclonal $\alpha \mathrm{CD}$ proteins is certainly due in part to the heterogeneity of their $\mathrm{N}$ terminal residues. It may also be related to two other features: the high carbohydrate content of most $\propto$ CD proteins and their high tendency to polymerize. The diagnosis is usually suspected or established by the immunoelectrophoretic analysis of the serum of these patients. In many cases, the protein abnormality escapes routine immunoelectrophoresis with polyvalent antisera to human normal serum. Analysis with monospecific antisera to $\operatorname{IgA}$ is an absolute need. The abnormal component usually gives an abnormal precipitin line either extending from the $\alpha 1$ globulins to the slow $\beta 2$ region or showing a faster electrophoretic mobility than normal IgA. The anomalous component does of course not precipitate with antisera to light chains. It should however be emphasized that this absence of precipitation is not a sufficient criterion for the diagnosis of $\alpha$ CD since many IgA myeloma proteins (mainly with $\lambda$ chains) fail to precipitate with most anti light chain antisera. The use of selected antisera to IgA containing antibodies related to the conformational specificity of the Fab region or of the immuno selection method were found to be useful for the diagnosis of $\alpha$ CD. In all doubtful cases, the pathological protein should be purified, reduced and alkylated and its lack of light chains should be directly demonstrated. To add to the difficulty of the diagnosis of $\alpha C D$, the amount of pathological protein in the urine was found to be small or minimal. In most patients however, the pathological protein was detected in the concentrated urine and had the some electrophoretic and immunochemical characteristics as in the serum. Bence-Jones proteinuria was never found. As expected in view of the involvement of the intestinal tract by the proliferative process in most patients with $\alpha \mathrm{CD}$, the pathological protein was found in significant amounts in jejunal fluid.

Alpha chain disease appears to be a condition affecting primarily the secretory IgA system and mainly the digestive tract. Its age distribution is in sharp contrast to that of multiple myeloma since $\alpha \mathrm{CD}$ occurs mainly in the second and third decade of life. Only 3 cases of $\alpha$ CD without detectable intestinal involvement have been reported and represent the respiratory form of the disease but the pathology of these cases is very poorly documented. Other non intestinal forms of $\alpha \mathrm{CD}$ (with involvement of salivary glands for instance) will probably be described in the future. In all 
other recognized cases we are dealing with the digestive form of the disease and the clinical features are strikingly uniform. The disease is usually revealed by chronic diarrhea, a severe malabsorption syndrome with considerable loss of weight, steatorrhea and hypocalcemia, and excessive fecal losses of water and electrolytes. Abdominal pains were often a major presenting symptom and abdominal masses were palpable in several patients. Finger clubbing was noted in many patients. Radiological studies and multiple intestinal biopsies showed that the whole length of the small intestine is usually involved. Rectal localization was detected by biopsy in several cases. When laparotomy was performed, diffuse mesenteric lymphadenopathies were often found. In contrast with the diffuse enteromesenteric lesions, the involvement of other organs (liver, spleen, peripheral nodes, marrow) is usually not observed or occurs at a late stage.

Pathological examination of the small intestine showed in all patients a diffuse and massive infiltration of the lamina propria with lymphoid cells. The cellular infiltrate was predominantly plasma cell in type; Secondary villous atrophy and crypt sparsity was noted in most instances, whereas the luminal epithelium showed relatively normal appearance. Sections of the mesenteric nodes may show a similar lymphoid and mainly plasmacytic proliferation. In many patients, the plasma cell infiltration in the lamina propria is moderately invasive and made of non dystrophic cells. However in a few cases, the neoplastic nature of the plasmacytic proliferation has been convincingly demonstrated. In many patients, who sometimes exhibit a tumoral symptomatology with possible intestinal obstruction, pathological features of truly malignant lymphoma were found in addition to the diffuse plasma cell proliferation. These sarcomas involved the gut, with often circumscribed tumors, or the mesenteric lymph nodes or both. These tumors show usually the pathological features of what has been recently called immunoblastic Iymphoma. These malignant sarcoma cells probably derive from the same $B$ cell clone as the underlying plasmacytic proliferation.

The evolution of $\alpha C D$ is most often progressive and fatal and the occurence of these truly malignant lymphomas represents probably a second stage of the disease. However complete clinical remission with disappearance of the $\alpha \mathrm{CD}$ protein and with a normal histological pattern has now been observed in several cases. It is of considerable interest that such an apparently complete remission was achieved in at least 4 patients who had been treated only with antibiotics per os. One of these patients is still in remission after a 5 years follow-up. It seems therefore reasonable to perform a laparotomy in all patients with $\alpha \mathrm{CD}$ and without overt malignancy in order to treat only with antibiotics and supportive therapy those patients in whom careful pathological examination disclosed plasma cell proliferation without obvious malignant sarcoma.

The geographic origin of patients affected with $\alpha$ CD is of special interest. Whereas the three patients with a respiratory form of the disease originated from Netherlands, United States and Great Britain, the common denonimator to the patients with intestinal involvement is that they originated from and had been living in areas with a high degree of infestation by intestinal micro-organisms: North Africa, South Africa, Middle East, Far East, South America or Southern Europe. This geographic distribution strongly suggests that enteral micro-organisms which provide a sustained local antigenic stimulus do play a role in the pathogenesis of 
$\alpha \mathrm{CD}$. These environmental factors could play a triggering role in the clonal proliferation. They may also be only predisposing factors leading to a non specific stimulation of immunocytes promoting the oncogenic effect of a virus. In any case the role of these environmental factors does not exclude the possible interference with predisposing genetic factors although the few family studies which have been performed have yielded essentially negative results. It is remarquable that the plasma cell proliferation resulting from the postulated antigenic stimulation appears to lead in almost all cases to heavy chain diseases and not to myeloma. Whether this is related to the nature of the triggered cells or to that of the triggering agents is presently a matter of speculation.

Since the non malignant nature of $\alpha \mathrm{CD}$ at its relatively early stage constitutes a real possibility, the reversibility of the hyperplastic process after administration of antibiotics and possibly withdrawal of the triggering agent is obviouly of considerable theoretical and practical interest. Thus $\alpha$ CD may well represent a model of a lymphoma charcterized by a continuous chain of events ranging from an apparently benign and reversible hyperplastic process to an overt neoplastic proliferation.

Authors' address: Prof. M. Seligmann, Laboratoire d'Immunochimie et d'Immunopathologie, Groupe INSERM. U. 108, Institut de Recherches sur les Maladies du Sang, Hôpital Saint Louis 2 place du Dr. Fournier, 75475 Paris Cedex 10, France. 